\title{
Augmented error system approach to control design for a class of neutral systems
}

\author{
Yujie $\mathrm{Xu}^{1}$, Jing Zhang ${ }^{1 *}$ and Fucheng Liao ${ }^{2}$
}

\author{
"Correspondence: \\ zhang1jing4@outlook.com \\ 1 Department of Basic Courses, \\ Beijing Union University, No. 97 \\ Beisihuan East Road, Chaoyang \\ District, Beijing, 100101, P.R. China \\ Full list of author information is \\ available at the end of the article
}

\begin{abstract}
In this paper, control design based on target tracking for a class of neutral systems is investigated by augmented error system approach. The dynamic equation of tracking error is derived by use of the desired output which is assumed to be known. Based on the main thought of preview control, the augmented error system is constructed. A criterion to guarantee the asymptotic stability of the corresponding nominal system is established. Through control design of the augmented error system, a delay-dependent control and a delay-independent control are respectively presented for the original neutral system. Control design in this paper contains integrators, which can effectively reduce static errors. Examples are given to illustrate the efficiency of the proposed method.
\end{abstract}

Keywords: neutral system; augmented error system; asymptotic stability; preview control; target tracking

\section{Introduction}

Time-delay is often encountered in many practical systems such as process control systems, neural network systems, and nuclear reactor systems (see $[1,2])$. It is well known that time-delay is a major source of instability and oscillation. Therefore, stability analysis and control design of time-delay systems are important in theory and practice (see [3-6]).

As a special class of time-delay systems, neutral systems contain time-delay both in the states and in the derivatives of states, which can reflect the systems' operation more comprehensively and accurately. It has been shown that a number of practical systems can be modeled by neutral systems such as heat exchangers, population ecology, and partial element equivalent circuit (PEEC) (see [7, 8]). Additionally, there exist some time-delay systems which can be transformed into neutral systems by model transformation, including lossless transmission model, standard delay systems, and standard distributed delay systems (see [9]). Due to the particularity of time-delay, stability issues of neutral systems are proved to be more complex. They have attracted much attention and hence a lot of research has been done over recent years (see $[10,11])$.

To the best of the authors' knowledge, though some frequency domain methods, such as the spectral decomposition theory and semi-group theory, are applied to investigate the stability of neutral systems, they are not as popular as Lyapunov-Krasovskii functional (LKF). Through LKF approach, many stability criteria have been proposed (see [11-13]). Recently, LMIs and free weighting matrices have been widely used in LKF for the stabil-

(c) $2015 \mathrm{Xu}$ et al. This article is distributed under the terms of the Creative Commons Attribution 4.0 International License (http://creativecommons.org/licenses/by/4.0/), which permits unrestricted use, distribution, and reproduction in any medium, provided you give appropriate credit to the original author(s) and the source, provide a link to the Creative Commons license, and indicate if changes were made. 
ity of neutral systems (see [14-16]). For example, by constructing a new LKF with free weighting matrices, stability criteria for uncertain neutral systems were investigated in [11]. Moreover, free weighting matrices and model transformations were both employed in [14] for cross terms. Then a less conservative stability criterion based on LMIs was derived for stochastic neutral systems. However, control design based on these stability criteria is usually the state feedback, which is delay-independent or delay-dependent.

Actually, it is required that the output should track the target in some control systems such as robot routine control systems. $H_{\infty}$ output tracking control, where the output of a control system tracks the output of a given reference model well in the sense, is often adopted as the main way of tracking control for neutral systems (see $[6,17,18]$ ). Augmented error system approach is usually used in tracking control by combining the error signal and the state. Sometimes, LKF uses augmented vector to simplify the results (see $[1,19])$.

This paper utilizes the known desired output, but not the reference model, to construct an augmented error system which contains the state vector, the error signal, the desired output, and their derivatives. Thus, control design in the paper contains not only state feedback, but also an integrator, which can effectively reduce static errors and is not similar to output tracking control (see [20,21]). The main thought comes from the theory of preview control, which belongs to tracking control. In this paper, we keep time-delay in the augmented error system, which is different from the ordinary method of preview control (see [20-22]).

Notations Throughout this paper, the following notations will be used. $\mathbf{R}^{n}$ denotes an $n$-dimensional Euclidean space. $\mathbf{R}^{n \times m}$ is the set of all $n \times m$ real matrices. $*$ refers to the symmetric part of a matrix. $P>0$ represents that $P$ is a symmetric positive definite matrix. $I$ denotes an identity matrix with appropriate dimensions.

\section{Problem statements}

Consider the following linear neutral system:

$$
\left\{\begin{array}{l}
\dot{x}(t)-G \dot{x}(t-h)=A x(t)+A_{1} x(t-h)+B u(t) \\
y(t)=C x(t), \quad t \geq 0 \\
x(t)=\phi(t), \quad t \in[-h, 0]
\end{array}\right.
$$

where $x(t) \in \mathbf{R}^{n}$ is the state vector, $u(t) \in \mathbf{R}^{m}$ is the input of system, and $y(t) \in \mathbf{R}^{p}$ is the output vector. $h>0$ denotes the constant time-delay, which appears in both the state and the derivative of state. The matrices $A \in \mathbf{R}^{n \times n}, A_{1} \in \mathbf{R}^{n \times n}, C \in \mathbf{R}^{p \times n}, G \in \mathbf{R}^{n \times n}, B \in \mathbf{R}^{n \times m}$ are known real constant matrices. And the initial condition $\phi(t)$ is a continuous vectorvalued function on $t \in[-h, 0]$. Define the operator $\mathcal{D} x(t)=x(t)-G x(t-h)$.

Let $r(t)$ be the $p \times 1$ desired output or the demand vector of system (1). Then the tracking error of system (1) is described as

$$
e(t)=y(t)-r(t)
$$

The basic problem considered in this paper is to design control for system (1) such that the output $y(t)$ tracks the desired output $r(t)$. For system (1), we assume the following. 
Assumption $1\left|\lambda_{i}(G)\right|<1(i=1,2, \ldots, n)$, where $\lambda_{i}(G)$ is the $i$ th characteristic value of $G$.

Assumption 2 The desired output $r(t) \in \mathbf{R}^{p}$ is piecewise differentiable with finite discontinuity points. Except its discontinuity points, $r(t)$ is differentiable up to the $s$ th order, i.e., $r^{\prime}(t), r^{\prime \prime}(t), \ldots, r^{(s-1)}(t)$ are all continuous and $r^{(s)}(t)=r^{(s+1)}(t)=\cdots=0$.

Remark 1 The stability of $\mathcal{D} x(t)$ can be replaced by the Schur-Cohn stability of $G$. It means that if all characteristic values of $G$ are in unite circle, the operator $\mathcal{D} x(t)$ is stable (see [9]). So Assumption 1 insures that $\mathcal{D} x(t)$ is stable.

Remark 2 System (1) is described by differential equations. So it is necessary that the derivatives of desired output are taken to construct an augmented error system. Assumption 2 can make sure the method is suitable for more extensive desired output such as staircase signal which is mostly common in signal processing (see [23]). Additionally, at discontinuity points of the desired output, we can take left or right derivatives as their derivatives for values of limited points do not affect the system's performance much.

Remark 3 If $r(t)$ is derivable for any order, its first to $s$ th derivatives are selected within error range, which is similar to the truncation method of signal procession (see [23]). Some desired output may be expanded into power series with limited terms if necessary.

The following well-known lemma will be used for providing the main results in the sequel.

Lemma 1 (Schur complement in [24]) Given the constant matrices $\Omega_{1}, \Omega_{2}, \Omega_{3}$, where $\Omega_{1}=$ $\Omega_{1}^{\mathrm{T}}$ and $0<\Omega_{2}=\Omega_{2}^{\mathrm{T}}$, then $\Omega_{1}+\Omega_{3}^{\mathrm{T}} \Omega_{2}^{-1} \Omega_{3}<0$ if and only if

$$
\left[\begin{array}{cc}
\Omega_{1} & \Omega_{3}^{\mathrm{T}} \\
\Omega_{3} & -\Omega_{2}
\end{array}\right]<0, \quad \text { or } \quad\left[\begin{array}{cc}
-\Omega_{2} & \Omega_{3} \\
\Omega_{3}^{\mathrm{T}} & \Omega_{1}
\end{array}\right]<0 \text {. }
$$

\section{Main results}

In this section, main results on both delay-dependent and delay-independent control design will be presented for system (1). Firstly, an augmented error system is constructed. And then, the stability criterion of its corresponding nominal system is proposed.

\subsection{Construction of an augmented error system}

In order to make better use of the desired output $r(t)$, we consider combining the tracking error $e(t)$ with system (1).

It follows from (2) that the dynamic equation of tracking error $e(t)$ is given by

$$
\dot{e}(t)-\alpha \dot{e}(t-h)=C \dot{x}(t)-\alpha C \dot{x}(t-h)-\dot{r}(t)+\alpha \dot{r}(t-h)
$$

where $0<\alpha<1$ is a constant. According to (1), we have

$$
\ddot{x}(t)-G \ddot{x}(t-h)=A \dot{x}(t)+A_{1} \dot{x}(t-h)+B \dot{u}(t) .
$$


Let $\delta r(t)=\dot{r}(t)-\dot{r}(t-h)$. Thus, by (1), (3) and (4), the augmented error system is obtained as follows:

$$
\dot{z}(t)-\bar{G} \dot{z}(t-h)=\bar{A}(t) z(t)+\bar{A}_{1}(t) z(t-h)+\bar{B} \dot{u}(t)+\bar{D} \delta r(t),
$$

where

$$
\begin{aligned}
& z(t)=\left[\begin{array}{c}
e(t) \\
x(t) \\
\dot{x}(t)
\end{array}\right], \quad \bar{G}=\left[\begin{array}{ccc}
\alpha I & 0 & 0 \\
0 & G & 0 \\
0 & 0 & G
\end{array}\right], \quad \bar{A}(t)=\left[\begin{array}{ccc}
0 & 0 & C \\
0 & 0 & I \\
0 & 0 & A
\end{array}\right], \\
& \bar{A}_{1}(t)=\left[\begin{array}{ccc}
0 & 0 & -\alpha C \\
0 & 0 & -G \\
0 & 0 & A_{1}
\end{array}\right], \quad \bar{B}=\left[\begin{array}{l}
0 \\
0 \\
B
\end{array}\right], \quad \bar{D}=\left[\begin{array}{c}
-I \\
0 \\
0
\end{array}\right] \text {. }
\end{aligned}
$$

In system (5), the tracking error $e(t)$ appears as a component of $z(t)$. And the state equation of $z(t)$ is related to the desired output $r(t)$. We wish to transfer system (5) into an ordinary neutral system. So we consider processing the desired output $r(t)$. Assume that $R(t)$ is the vector which contains the available desired output and its first to $s$ th order derivatives, namely

$$
R(t)=\left[r(t)^{\mathrm{T}}, r^{\prime}(t)^{\mathrm{T}}, \ldots, r^{(s-2)}(t)^{\mathrm{T}}, r^{(s-1)}(t)^{\mathrm{T}}\right]^{\mathrm{T}} .
$$

It follows from Assumption 2 that $R(t)$ satisfies $\dot{R}(t)=E R(t), \dot{R}(t-h)=E R(t-h)$, where

$$
E=\left[\begin{array}{ccccc}
0 & I_{p} & 0 & \cdots & 0 \\
0 & 0 & I_{p} & \cdots & 0 \\
\vdots & \vdots & \vdots & \ddots & \vdots \\
0 & 0 & 0 & \cdots & I_{p} \\
0 & 0 & 0 & \cdots & 0
\end{array}\right] .
$$

Denote $X(t)=\left[z(t)^{\mathrm{T}}, R(t)^{\mathrm{T}}\right]^{\mathrm{T}}$, system (5) can be rewritten as

$$
\dot{X}(t)-\tilde{G} \dot{X}(t-h)=\tilde{A} X(t)+\tilde{A}_{1} X(t-h)+\tilde{B} \dot{u}(t),
$$

where

$$
\begin{aligned}
& \tilde{G}=\left[\begin{array}{cc}
\bar{G} & 0 \\
0 & \alpha I
\end{array}\right], \quad \tilde{A}=\left[\begin{array}{cc}
\bar{A} & D_{R} \\
0 & E
\end{array}\right], \quad \tilde{A}_{1}=\left[\begin{array}{cc}
\bar{A}_{1} & -\alpha D_{R} \\
0 & -\alpha E
\end{array}\right], \quad \tilde{B}=\left[\begin{array}{c}
\bar{B} \\
0
\end{array}\right], \\
& D_{R}=[0, \bar{D}, 0, \ldots, 0] .
\end{aligned}
$$

Therefore, the problem tackled in this paper is to design control for system (6).

\subsection{Asymptotic stability analysis of the nominal system}

The nominal system of (6) is

$$
\dot{X}(t)-\tilde{G} \dot{X}(t-h)=\tilde{A} X(t)+\tilde{A}_{1} X(t-h) .
$$


Now a criterion is presented for the asymptotic stability of system (7).

Theorem 1 System (7) is asymptotically stable if there exist $P>0$ and $Q>0$ such that the following symmetric matrix inequality holds:

$$
\left[\begin{array}{cccc}
P \tilde{A}+\tilde{A}^{\mathrm{T}} P & P \tilde{A} \tilde{G} & P \tilde{A}_{1} & I \\
* & -Q / 2 & 0 & \tilde{G}^{\mathrm{T}} \\
* & * & -Q / 2 & 0 \\
* & * & * & -Q^{-1}
\end{array}\right]<0 .
$$

Proof Based on Assumption 1 and $0<\alpha<1$, it is easy to get $\left|\lambda_{i}(\bar{G})\right|<1(i=1,2, \ldots, 2 n+p)$. Therefore, we conclude that $\left|\lambda_{j}(\tilde{G})\right|<1(j=1,2, \ldots, 2 n+p+s p)$, which ensures $\mathcal{D} X(t)=$ $X(t)-\tilde{G} X(t-h)$ is stable (see [9]).

Consider the following Lyapunov functional:

$$
V(t)=V_{1}(t)+V_{2}(t)
$$

where $V_{1}(t)=\mathcal{D} X(t)^{\mathrm{T}} P \mathcal{D} X(t), V_{2}(t)=\int_{-h}^{0} X(t+\theta)^{\mathrm{T}} Q X(t+\theta) \mathrm{d} \theta$, the matrices $P=P^{\mathrm{T}}>0$ and $Q=Q^{\mathrm{T}}>0$ are to be determined.

Taking time derivatives of $V_{1}(t)$ and $V_{2}(t)$ along system (7) yields

$$
\begin{aligned}
\dot{V}_{1}(t)= & {[\dot{X}(t)-\tilde{G} \dot{X}(t-h)]^{\mathrm{T}} P \mathcal{D} X(t)+\mathcal{D} X(t)^{\mathrm{T}} P[\dot{X}(t)-\tilde{G} \dot{X}(t-h)] } \\
= & {\left[\tilde{A} X(t)+\tilde{A}_{1} X(t-h)\right]^{\mathrm{T}} P[X(t)-\tilde{G} X(t-h)] } \\
& +[X(t)-\tilde{G} X(t-h)]^{\mathrm{T}} P\left[\tilde{A} X(t)+\tilde{A}_{1} X(t-h)\right] \\
= & X(t)^{\mathrm{T}}\left(\tilde{A}^{\mathrm{T}} P+P \tilde{A}\right) X(t)+X(t)^{\mathrm{T}}\left(-\tilde{A}^{\mathrm{T}} P \tilde{G}+P \tilde{A}_{1}\right) X(t-h) \\
& +X(t-h)^{\mathrm{T}}\left(\tilde{A}_{1}^{\mathrm{T}} P-\tilde{G}^{\mathrm{T}} P \tilde{A}\right) X(t) \\
& +X(t-h)^{\mathrm{T}}\left(-\tilde{A}_{1}^{\mathrm{T}} P \tilde{G}-\tilde{G}^{\mathrm{T}} P \tilde{A}_{1}\right) X(t-h), \\
\dot{V}_{2}(t)= & X(t)^{\mathrm{T}} Q X(t)-X(t-h)^{\mathrm{T}} Q X(t-h) .
\end{aligned}
$$

Thus we get

$$
\begin{aligned}
\dot{V}(t)= & X(t)^{\mathrm{T}}\left(\tilde{A}^{\mathrm{T}} P+P \tilde{A}+Q\right) X(t)+X(t)^{\mathrm{T}}\left(P \tilde{A}_{1}-\tilde{A}^{\mathrm{T}} P \tilde{G}\right) X(t-h) \\
& +X(t-h)^{\mathrm{T}}\left(\tilde{A}_{1}^{\mathrm{T}} P-\tilde{G}^{\mathrm{T}} P \tilde{A}\right) X(t) \\
& +X(t-h)^{\mathrm{T}}\left(-\tilde{A}_{1}^{\mathrm{T}} P \tilde{G}-\tilde{G}^{\mathrm{T}} P \tilde{A}_{1}-Q\right) X(t-h) .
\end{aligned}
$$

Let $M=\frac{Q}{2}-\tilde{G}^{\mathrm{T}} Q \tilde{G}$. In view of the definition of $\mathcal{D}$, the derivative of $V$ with respect to $t$ can be rewritten as

$$
\begin{aligned}
\dot{V}(t)= & \mathcal{D} X(t)^{\mathrm{T}}\left(P \tilde{A}+\tilde{A}^{\mathrm{T}} P+Q\right) \mathcal{D} X(t)+2 \mathcal{D} X(t)^{\mathrm{T}}(Q+P \tilde{A}) \tilde{G} X(t-h) \\
& +2 \mathcal{D} X(t)^{\mathrm{T}} P \tilde{A}_{1} X(t-h)-X(t-h)^{\mathrm{T}}\left(M+\frac{Q}{2}\right) X(t-h) \\
= & \mathcal{D} X(t)^{\mathrm{T}}\left[P \tilde{A}+\tilde{A}^{\mathrm{T}} P+Q+(Q+P \tilde{A}) \tilde{G} M^{-1} \tilde{G}^{\mathrm{T}}(Q+P \tilde{A})^{\mathrm{T}}\right.
\end{aligned}
$$




$$
\begin{aligned}
& \left.+2 P \tilde{A}_{1} Q^{-1} \tilde{A}_{1}^{\mathrm{T}} P\right] \mathcal{D} X(t)-\left[X(t-h)^{\mathrm{T}}-\mathcal{D} X(t)^{\mathrm{T}}(Q+P \tilde{A}) \tilde{G} M^{-1}\right] \\
& \times M\left[X(t-h)-M^{-1} \tilde{G}^{\mathrm{T}}(Q+P \tilde{A})^{\mathrm{T}} \mathcal{D} X(t)\right] \\
& -\left[X(t-h)^{\mathrm{T}}-2 \mathcal{D} X(t)^{\mathrm{T}} P \tilde{A}_{1} Q^{-1}\right] \frac{Q}{2}\left[X(t-h)-2 Q^{-1} \tilde{A}_{1}^{\mathrm{T}} P \mathcal{D} X(t)\right] .
\end{aligned}
$$

Since $Q=Q^{\mathrm{T}}>0$, we obtain that $\dot{V}<0$ if the following inequalities

$$
M=\frac{Q}{2}-\tilde{G}^{\mathrm{T}} Q \tilde{G}>0
$$

and

$$
P \tilde{A}+\tilde{A}^{\mathrm{T}} P+Q+(Q+P \tilde{A}) \tilde{G} M^{-1} \tilde{G}^{\mathrm{T}}(Q+P \tilde{A})^{\mathrm{T}}+2 P \tilde{A}_{1} Q \tilde{A}_{1}^{\mathrm{T}} P<0
$$

are feasible. Then, according to Lyapunov stability theory, system (7) is asymptotically stable.

By Schur complement, (9) and (10), we get

$$
\left[\begin{array}{cc}
P \tilde{A}+\tilde{A}^{\mathrm{T}} P+Q+2 P \tilde{A}_{1} Q \tilde{A}_{1}^{\mathrm{T}} P & (Q+P \tilde{A}) \tilde{G} \\
* & -M
\end{array}\right]<0 .
$$

In view of

$$
\begin{aligned}
& {\left[\begin{array}{cc}
P \tilde{A}+\tilde{A}^{\mathrm{T}} P+Q+2 P \tilde{A}_{1} Q^{-1} \tilde{A}_{1}^{\mathrm{T} P} & (Q+P \tilde{A}) \tilde{G} \\
* & -M
\end{array}\right]} \\
& \quad=\left[\begin{array}{cc}
P \tilde{A}+\tilde{A}^{\mathrm{T}} P+Q & (Q+P \tilde{A}) \tilde{G} \\
* & -Q / 2+\tilde{G}^{\mathrm{T}} Q \tilde{G}
\end{array}\right]+\left[\begin{array}{c}
P \tilde{A}_{1} \\
0
\end{array}\right]\left(2 Q^{-1}\right)\left[\begin{array}{cc}
\tilde{A}_{1}^{\mathrm{T}} P & 0
\end{array}\right]
\end{aligned}
$$

and $Q>0,(11)$ is equivalent to

$$
\left[\begin{array}{ccc}
P \tilde{A}+\tilde{A}^{\mathrm{T}} P+Q & (Q+P \tilde{A}) \tilde{G} & P \tilde{A}_{1} \\
* & -Q / 2+\tilde{G}^{\mathrm{T}} Q \tilde{G} & 0 \\
* & * & -Q / 2
\end{array}\right]<0 .
$$

On the other hand,

$$
\begin{aligned}
& {\left[\begin{array}{ccc}
P \tilde{A}+\tilde{A}^{\mathrm{T}} P+Q & (Q+P \tilde{A}) \tilde{G} & P \tilde{A}_{1} \\
* & -Q / 2+\tilde{G}^{\mathrm{T}} Q \tilde{G} & 0 \\
* & * & -Q / 2
\end{array}\right]} \\
& =\left[\begin{array}{ccc}
P \tilde{A}+\tilde{A}^{\mathrm{T}} P & P \tilde{A} \tilde{G} & P \tilde{A}_{1} \\
* & -Q / 2 & 0 \\
* & * & -Q / 2
\end{array}\right]+\left[\begin{array}{c}
I \\
\tilde{G}^{\mathrm{T}} \\
0
\end{array}\right] Q\left[\begin{array}{lll}
I & \tilde{G} & 0
\end{array}\right] .
\end{aligned}
$$

So inequality (12) equals

$$
\left[\begin{array}{ccc}
P \tilde{A}+\tilde{A}^{\mathrm{T}} P & P \tilde{A} \tilde{G} & P \tilde{A}_{1} \\
* & -Q / 2 & 0 \\
* & * & -Q / 2
\end{array}\right]+\left[\begin{array}{c}
I \\
\tilde{G}^{\mathrm{T}} \\
0
\end{array}\right] Q\left[\begin{array}{lll}
I & \tilde{G} & 0
\end{array}\right]<0
$$


By Lemma 1, the above inequality implies that (8) holds.

As a foundation, Theorem 1 provides a sufficient condition for the asymptotic stability of system (7). In the following sections, the proposed delay-independent and delaydependent controllers are calculated not only to stabilize the closed-loop neutral system, but also to fully utilize the desired output $r(t)$.

\subsection{Delay-independent control for system (1)}

Suppose $\dot{u}(t)=K X(t)$ is a delay-independent control for system (6), where $K \in \mathbf{R}^{1 \times[2 n+(s+1) p]}$ is the feedback gain matrix. Substituting $\dot{u}(t)=K X(t)$ into system (6), we obtain the following closed-loop system:

$$
\dot{X}(t)-\tilde{G} \dot{X}(t-h)=(\tilde{A}+\tilde{B} K) X(t)+\tilde{A}_{1} X(t-h)
$$

By using Schur complement and replacing $\tilde{A}$ in system (7) with $\tilde{A}+\tilde{B} K$, we have the following theorem.

Theorem 2 Suppose Assumption 1 holds for system (1). The closed-loop system of (6) is asymptotically stable if there exist matrices $P>0, Q>0$ and $K$ such that

$$
\left[\begin{array}{cccc}
Z+Z^{\mathrm{T}} & Z \tilde{G} & P \tilde{A}_{1} & I \\
* & -Q / 2 & 0 & \tilde{G}^{\mathrm{T}} \\
* & * & -Q / 2 & 0 \\
* & * & * & -Q^{-1}
\end{array}\right]<0
$$

where $Z=P \tilde{A}+P \tilde{B} K$. Then $\dot{u}(t)=K X(t)$ is a delay-independent control for system (6).

Considering the structure of $X(t)$ in (6), $K$ can be decomposed as $K=\left[k_{1}, k_{2}, k_{3}, k_{4}\right]$. Therefore, $\dot{u}(t)$ for system (6) can be expressed as

$$
\dot{u}(t)=k_{1} e(t)+k_{2} x(t)+k_{3} \dot{x}(t)+\sum_{j=0}^{s-1} k_{4, j} r^{(j)}(t),
$$

where $k_{4}=\left[k_{4,0}, k_{4,1}, \ldots, k_{4, s-1}\right]$. So

$$
\begin{aligned}
u(t)-u(-h)= & k_{1} \int_{-h}^{t} e(v) \mathrm{d} v+k_{2} \int_{-h}^{t} x(v) \mathrm{d} v+k_{3} \int_{-h}^{t} \dot{x}(v) \mathrm{d} v \\
& +k_{4,0} \int_{0}^{t} r(v) \mathrm{d} v+\sum_{j=1}^{s-1} k_{4, j} \int_{-h}^{t} r^{(j-1)}(v) \mathrm{d} v .
\end{aligned}
$$

Then control $u(t)$ of system (1) is provided in the following theorem. 
Theorem 3 Suppose Assumption 1 holds for system (1). If there exists a matrix K satisfying (14), the delay-independent control for system (1) can be expressed as

$$
\begin{aligned}
u(t)= & \left(k_{1} C+k_{2}\right) \int_{-h}^{0} \phi(v) \mathrm{d} v+k_{1} \int_{0}^{t} e(v) \mathrm{d} v+k_{2} \int_{0}^{t} x(v) \mathrm{d} v+k_{3} x(t) \\
& -k_{3} \phi(-h)+k_{4,0} \int_{0}^{t} r(v) \mathrm{d} v+\sum_{j=1}^{s-1} k_{4, j} r^{(j-1)}(t)
\end{aligned}
$$

where $K=\left[k_{1}, k_{2}, k_{3}, k_{4}\right], k_{4}=\left[k_{4,0}, k_{4,1}, \ldots, k_{4, s-1}\right]$.

Remark 4 From (15), we can find that control $u(t)$, which is calculated from $\dot{u}(t)$, not only contains state feedback and the integral of state vector $x(t)$, but also relates to the initial condition $\phi(t)$. Furthermore, both the integrator on tracking error signal $e(t)$ and the integral of desired output $r(t)$ are contained in control $u(t)$. It is well known that introduction of integrators to control design is helpful to eliminate static errors (see [21]).

\subsection{Delay-dependent control for system (1)}

Now we suppose that $\dot{u}(t)=L X(t)+W X(t-h)$ is a delay-dependent control for system (6), where $L \in \mathbf{R}^{1 \times[2 n+(s+1) p]}$ and $W \in \mathbf{R}^{1 \times[2 n+(s+1) p]}$ are the feedback gain matrices. Substituting the control into system (6), we obtain the closed-loop system as follows:

$$
\dot{X}(t)-\tilde{G} \dot{X}(t-h)=(\tilde{A}+\tilde{B} L) X(t)+\left(\tilde{A}_{1}+\tilde{B} W\right) X(t-h) .
$$

From Theorem 1 , replacing $\tilde{A}$ and $\tilde{A}_{1}$ of system (7) respectively with $\tilde{A}+\tilde{B} L$ and $\tilde{A}_{1}+\tilde{B} W$ presents the following theorem to guarantee the asymptotic stability of system (16).

Theorem 4 Suppose Assumption 1 holds for (1). System (16) is asymptotically stable if there exist matrices $P>0, Q>0, L$ and $W$ such that

$$
\left[\begin{array}{cccc}
Z+Z^{\mathrm{T}} & Z \tilde{G} & Y & I \\
* & -Q / 2 & 0 & \tilde{G}^{\mathrm{T}} \\
* & * & -Q / 2 & 0 \\
* & * & * & -Q^{-1}
\end{array}\right]<0,
$$

where $Z=P \tilde{A}+P \tilde{B} L, Y=P \tilde{A}_{1}+P \tilde{B} W$. Then $\dot{u}(t)=L X(t)+W X(t-h)$ is a delay-dependent control for system (16).

If there exist matrices $L$ and $W$ to satisfy Theorem $4, L$ and $W$ are decomposed as $L=$ $\left[l_{1}, l_{2}, l_{3}, l_{4}\right], W=\left[w_{1}, w_{2}, w_{3}, w_{4}\right]$. Then control $\dot{u}(t)$ of system $(6)$ is deduced as

$$
\begin{aligned}
\dot{u}(t)= & l_{1} e(t)+l_{2} x(t)+l_{3} \dot{x}(t)+\sum_{j=0}^{s-1} l_{4, j} r^{(j)}(t) \\
& +w_{1} e(t-h)+w_{2} x(t-h)+w_{3} \dot{x}(t-h)+\sum_{j=0}^{s-1} w_{4, j} r^{(j)}(t-h),
\end{aligned}
$$


where $l_{4}=\left[l_{4,0}, l_{4,1}, \ldots, l_{4, s-1}\right], w_{4}=\left[w_{4,0}, w_{4,1}, \ldots, w_{4, s-1}\right]$. So

$$
\begin{aligned}
u(t)-u(-h)= & l_{1} \int_{-h}^{t} e(v) \mathrm{d} v+l_{2} \int_{-h}^{t} x(v) \mathrm{d} v+l_{3} \int_{-h}^{t} \dot{x}(v) \mathrm{d} v \\
& +\sum_{j=0}^{s-1} l_{4, j} \int_{-h}^{t} r^{(j-1)}(v) \mathrm{d} v+w_{1} \int_{-h}^{t} e(v-h) \mathrm{d} v \\
& +w_{2} \int_{-h}^{t} x(v-h) \mathrm{d} v+w_{3} \int_{-h}^{t} \dot{x}(v-h) \mathrm{d} v \\
& +\sum_{j=0}^{s-1} w_{4, j} \int_{-h}^{t} r^{(j)}(v-h) \mathrm{d} v .
\end{aligned}
$$

Using variable substitution to the integral terms in which the integrand functions are related to time-delay, we have

$$
\begin{aligned}
u(t)-u(-h)= & l_{1} \int_{-h}^{t} e(v) \mathrm{d} v+l_{2} \int_{-h}^{t} x(v) \mathrm{d} v+l_{3} \int_{-h}^{t} \dot{x}(v) \mathrm{d} v \\
& +\sum_{j=0}^{s-1} l_{4, j} \int_{-h}^{t} r^{(j)}(v) \mathrm{d} v+w_{1} \int_{-2 h}^{t-h} e(\theta) \mathrm{d} \theta+w_{2} \int_{-2 h}^{t-h} x(\theta) \mathrm{d} \theta \\
& +w_{3} \int_{-2 h}^{t-h} \dot{x}(\theta) \mathrm{d} \theta+\sum_{j=0}^{s-1} w_{4, j} \int_{-2 h}^{t-h} r^{(j)}(\theta) \mathrm{d} \theta .
\end{aligned}
$$

Commonly, time-delay $h$ is much smaller than the system's run time. So the control law of $t \leq h$ has little effect on the system's whole running status. Therefore, we only consider the control law when $t>h$. For $t-h>0$, it is not difficult to obtain that

$$
\begin{aligned}
& w_{1} \int_{-2 h}^{t-h} e(\theta) \mathrm{d} \theta=w_{1} C \int_{-h}^{0} \phi(\theta) \mathrm{d} \theta+w_{1} \int_{0}^{t-h} e(\theta) \mathrm{d} \theta, \\
& w_{2} \int_{-2 h}^{t-h} x(\theta) \mathrm{d} \theta=w_{2} \int_{-h}^{0} \phi(\theta) \mathrm{d} \theta+w_{2} \int_{0}^{t-h} x(\theta) \mathrm{d} \theta, \\
& w_{3} \int_{-2 h}^{t-h} \dot{x}(\theta) \mathrm{d} \theta=w_{3}(x(t-h)-\phi(-h)), \\
& \sum_{j=0}^{s-1} w_{4, j} \int_{-2 h}^{t-h} r^{(j)}(\theta) \mathrm{d} \theta=w_{4,0} \int_{0}^{t-h} r(v) \mathrm{d} v+\sum_{j=1}^{s-1} w_{4, j} r^{(j-1)}(t-h) .
\end{aligned}
$$

Then the control based on target tracking is presented as the following theorem.

Theorem 5 Suppose Assumption 1 holds for system (1). If there exist matrices $L$ and $W$ satisfying (17), the delay-dependent control for system (1) can be expressed as

$$
\begin{aligned}
u(t)= & \left(l_{1} C+w_{1} C+l_{2}+w_{2}\right) \int_{-h}^{0} \phi(\theta) \mathrm{d} \theta-\left(l_{3}+w_{3}\right) \phi(-h)+l_{3} x(t) \\
& +w_{3} x(t-h)+l_{1} \int_{0}^{t} e(\theta) \mathrm{d} \theta+w_{1} \int_{0}^{t-h} e(\theta) \mathrm{d} \theta+l_{2} \int_{0}^{t} x(\theta) \mathrm{d} \theta
\end{aligned}
$$




$$
\begin{aligned}
& +w_{2} \int_{0}^{t-h} x(\theta) \mathrm{d} \theta+w_{4,0} \int_{0}^{t-h} r(\theta) \mathrm{d} \theta+l_{4,0} \int_{0}^{t} r(\theta) \mathrm{d} \theta \\
& +\sum_{j=1}^{s-1}\left[w_{4, j} r^{(j-1)}(t-h)+l_{4, j} r^{(j-1)}(t)\right] .
\end{aligned}
$$

Remark 5 Compared with (15), control design in (18) contains more information to help the output $y(t)$ track the desired output $r(t)$ effectively.

\section{Examples}

In this section, two examples are given to show the effectiveness of the proposed control design method.

Example 1 Consider system (1) with the following parameters:

$$
\begin{aligned}
& G=\left[\begin{array}{cc}
0.2 & -0.01 \\
0 & 0.01
\end{array}\right], \quad A=\left[\begin{array}{cc}
1 & 0.6 \\
0 & -1
\end{array}\right], \quad A_{1}=\left[\begin{array}{cc}
0.03 & -0.2 \\
0.2 & 0.1
\end{array}\right], \quad B=\left[\begin{array}{l}
0.8 \\
0.4
\end{array}\right], \\
& C=\left[\begin{array}{ll}
1 & 1
\end{array}\right], \quad h=0.2, \quad \phi(\theta)=\left[\begin{array}{c}
0.2 \theta \\
(-1)^{[\theta]}
\end{array}\right], \quad-h<\theta \leq 0 .
\end{aligned}
$$

Assume the desired output $r(t)=1 / t$. Obviously, the desired output is derivable for any order. By Remark 3, we choose $s=3$. And in the dynamic equation of $e(t)$, we choose $\alpha=0.5$.

Case 1: Control design independent of time-delay is firstly considered. By Theorem 3, the feedback gain matrix $K$ is

$$
\left[\begin{array}{llllllll}
-6.8292 & -6.8126 & 5.5366 & -6.2757 & -5.3731 & -1.6606 & 1.4060 & 0.6992
\end{array}\right] \text {. }
$$

Furthermore, the corresponding state curve and output response curve are shown respectively in Figures 1 and 2.

Choose $E=0$ and $D_{R}=0$ when constructing the augmented error system, i.e., remove the desired output $r(t)$ from control $u(t)$ of Theorem 3 . Then we get the control irrelevant to target tracking and time delay. The state curve and output response curve are shown respectively as Figures 3 and 4.

From Figures 1-4, we find that when the control is based on target tracking, the state curve and the output response are much smoother, and the curve amplitude is much smaller.

Case 2: We observe the effect of control design dependent of time-delay. By Theorem 4, feedback gain matrices $L$ and $W$ are respectively

$$
\begin{aligned}
& {[-0.0010,0.0002,0.0002,-1.1433,-0.1728,0.0002,0.0015,-0.0001]} \\
& {[0,0,0,-0.1291,0.1510,0,-0.0014,-0.0001]}
\end{aligned}
$$

Then the corresponding state curve and output response curve are shown respectively in Figures 5 and 6. 


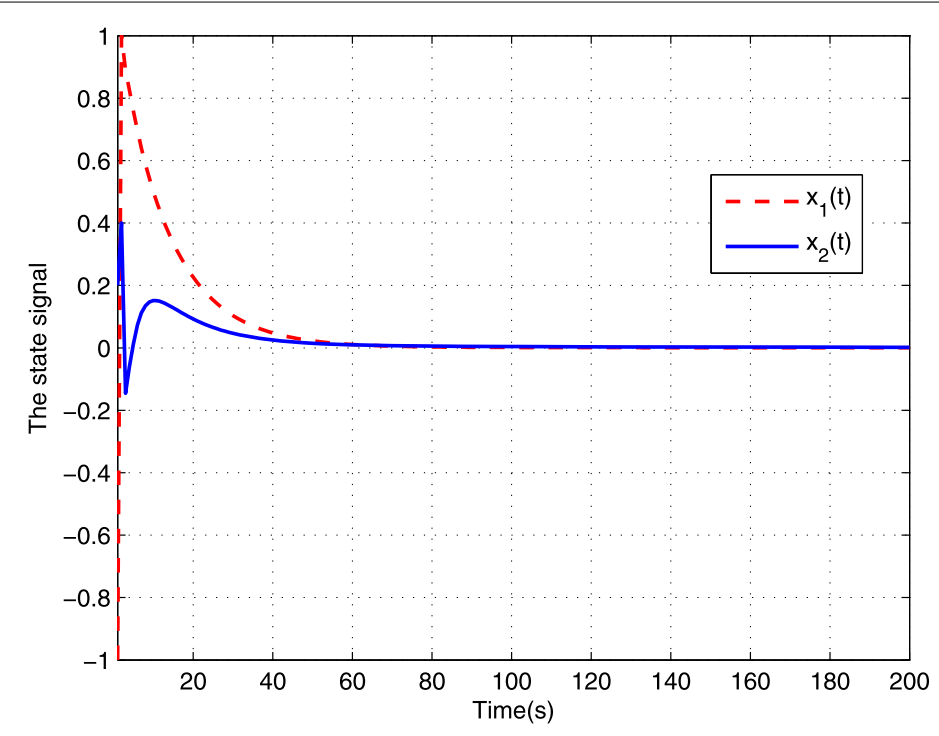

Figure 1 State curve of system (1) under delay-independent control based on target tracking.

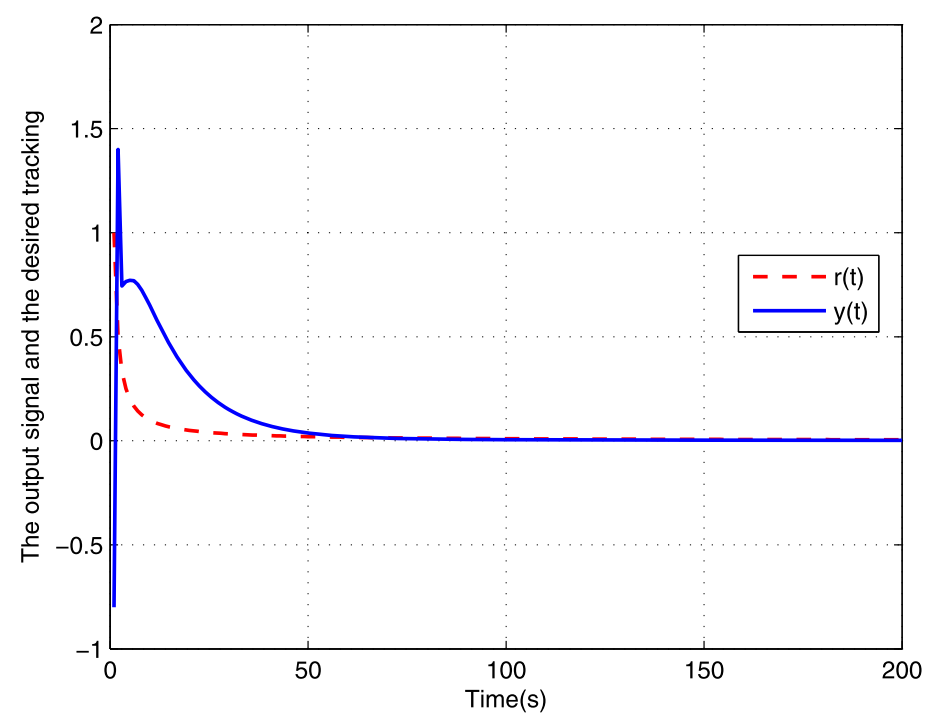

Figure 2 Output response of system (1) under delay-independent control based on target tracking.

When the control is relevant to time-delay but irrelevant to the desired output, the state curve and output response curve are shown respectively in Figures 7 and 8.

Comparing these figures, we find that for this system, the amplitude of the state curve and output response is much smaller when the control $u(t)$ is relevant to time delay.

Example 2 The following system comes from a small test circuit example which consists of a partial element equivalent circuit (PEEC) (see [8]). Based on model (1) of [8], it is given 


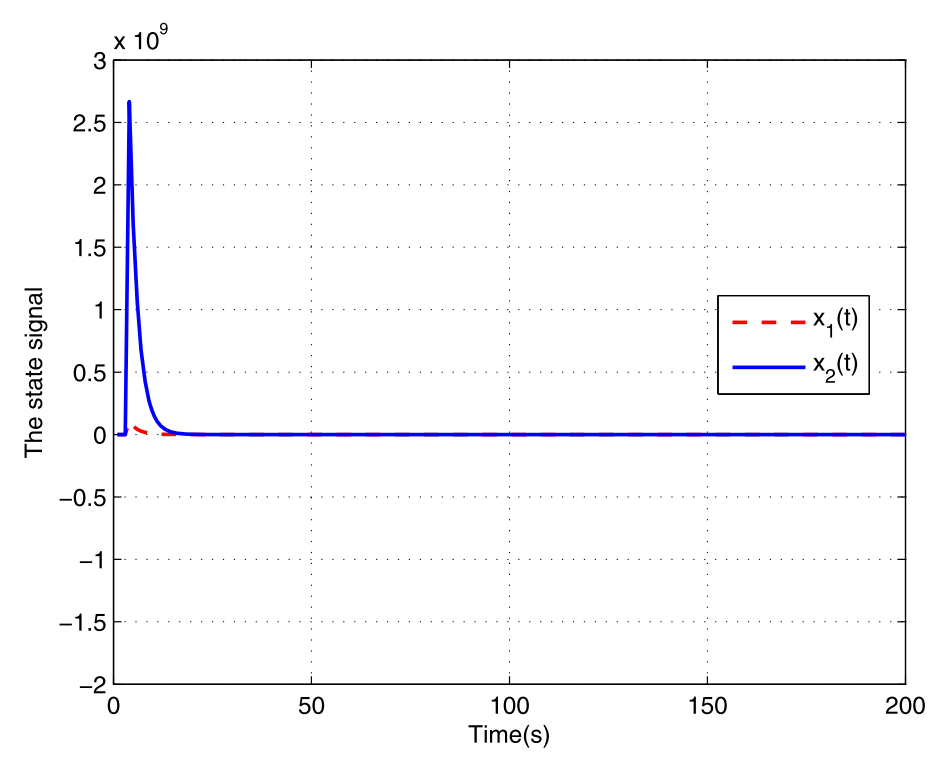

Figure 3 State curve of system (1) under delay-independent control without target tracking.

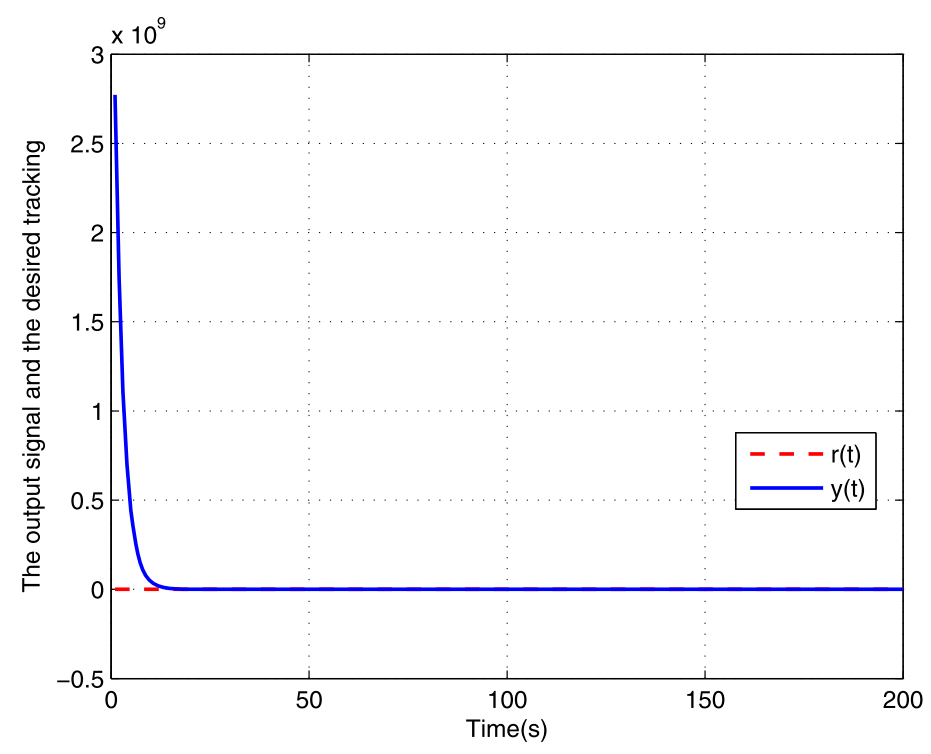

Figure 4 Output response of system (1) under delay-independent control without target tracking.

by

$$
\left\{\begin{array}{l}
\dot{x}(t)-\left[\begin{array}{cc}
0.01 & -0.5 \\
0 & 0.02
\end{array}\right] \dot{x}(t-0.2)=\left[\begin{array}{cc}
0.03 & -0.2 \\
0.25 & 0.2
\end{array}\right] x(t-h)+\left[\begin{array}{cc}
1 & 0.2 \\
0 & -1.5
\end{array}\right] x(t)+\left[\begin{array}{c}
1 \\
-2
\end{array}\right] u(t) \\
y(t)=\left[\begin{array}{ll}
1 & 2.5
\end{array}\right] x(t), \quad t \geq 0 \\
x(t)=\left[\begin{array}{c}
-t / 10 \\
\sin t
\end{array}\right], \quad t \in[-0.2,0]
\end{array}\right.
$$




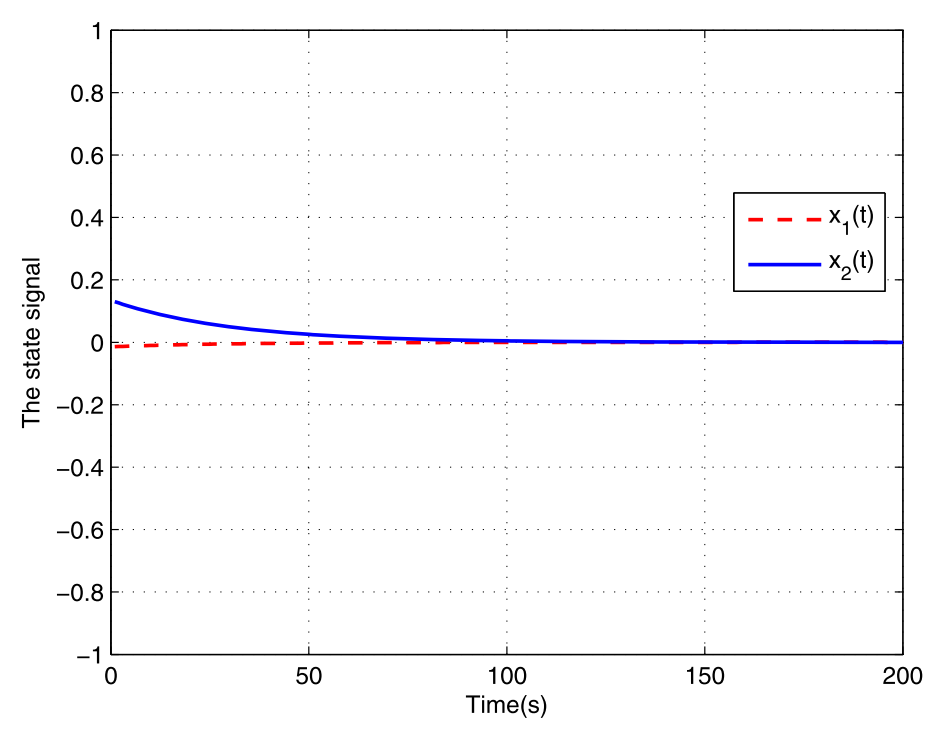

Figure 5 State curve of system (1) under delay-dependent control based on target tracking.

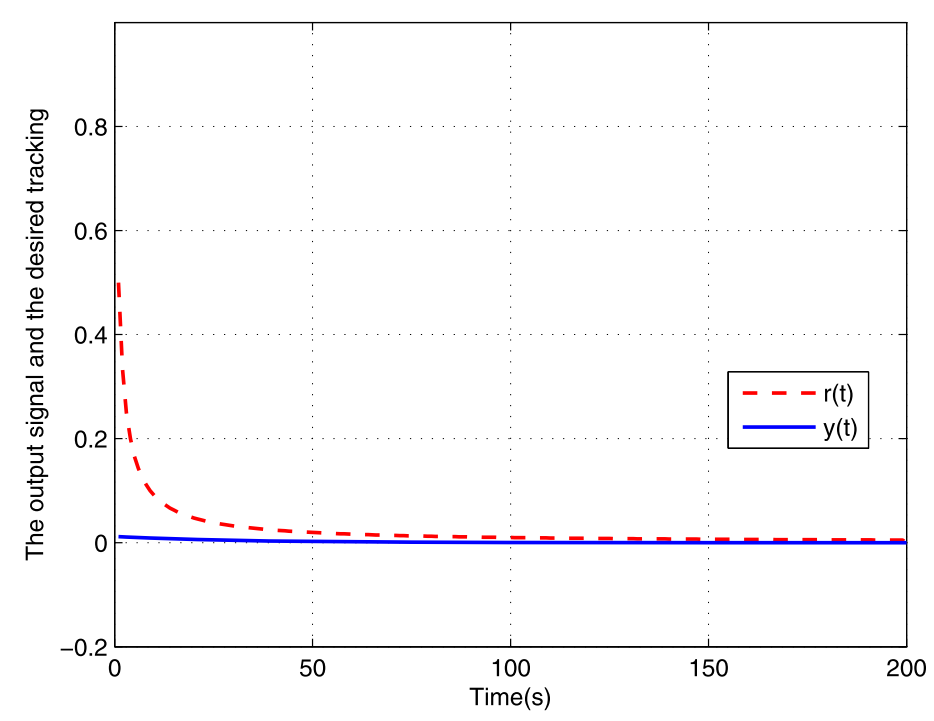

Figure 6 Output response of system (1) under delay-dependent control based on target tracking.

The desired output is assumed to be the following stair-step function:

$$
r(t)= \begin{cases}0, & t<40 \\ \frac{1}{40}(t-40), & 40 \leq t<80 \\ 1, & t \geq 80\end{cases}
$$

And in the dynamic equation of $e(t)$, we choose $\alpha=0.9$.

In this example, we only observe the control design based on target-tracking for system (19). By Theorem 3, we get that the state and the output response of system (19) under delay-independent control design are respectively shown as Figures 9 and 10. Then, by 


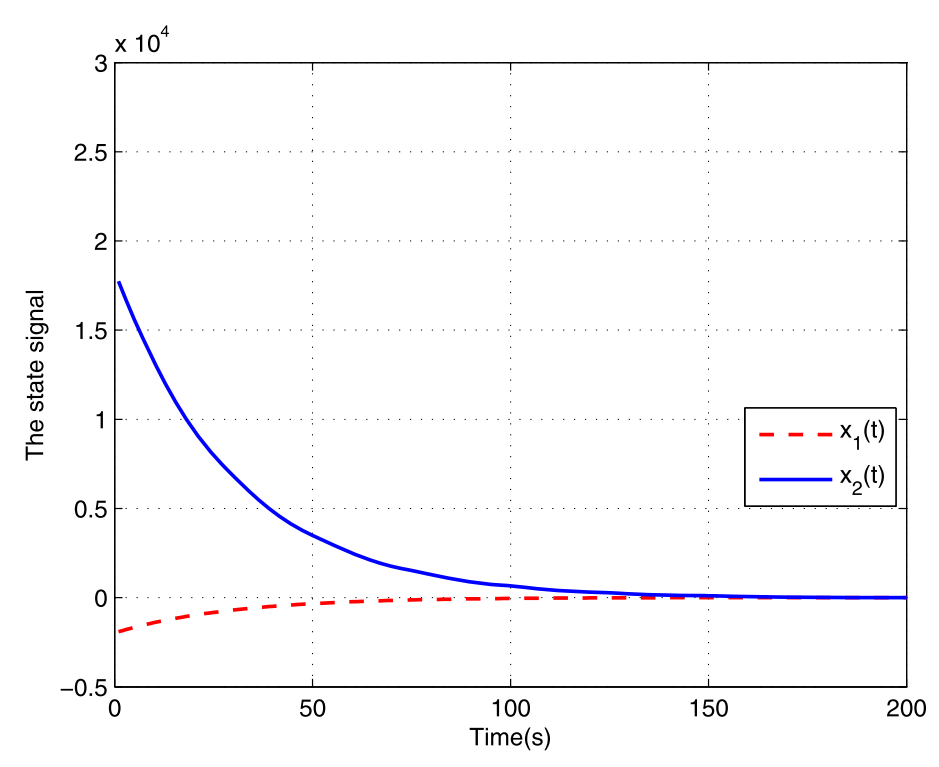

Figure 7 State curve of system (1) under delay-dependent control without target tracking.

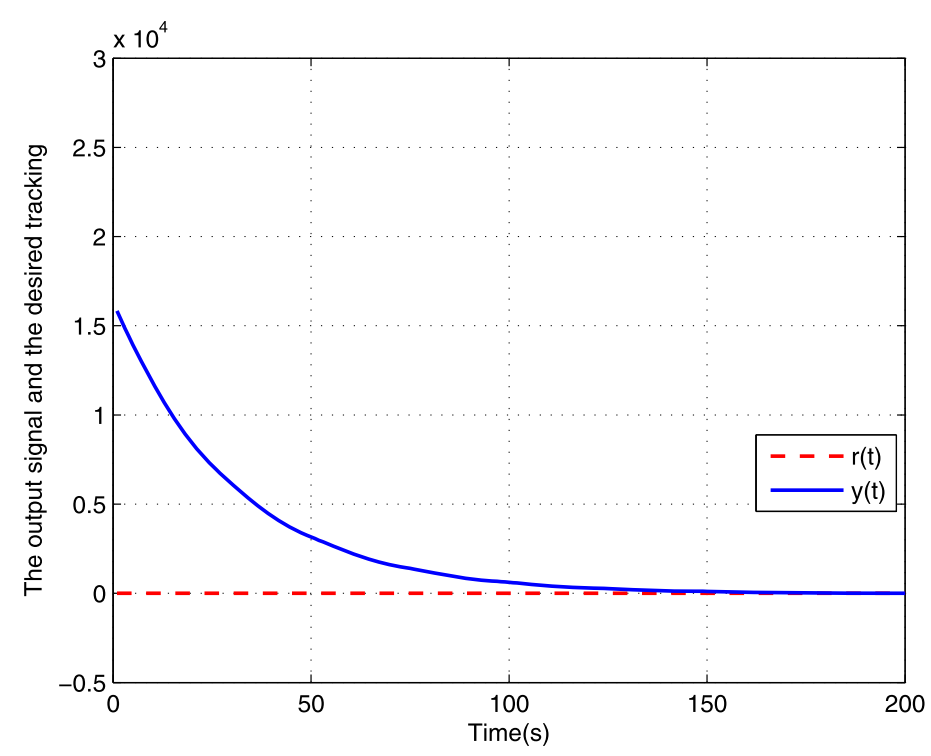

Figure 8 Output response of system (1) under delay-dependent control without target tracking.

Theorem 5, the corresponding curves under delay-dependent control design are shown in Figures 11 and 12.

Remark 6 The model in Example 2 comes from partial element equivalent circuits. In [8], the neutral system was described with zero output, whereas an output equation is added to the system in Example 2. 


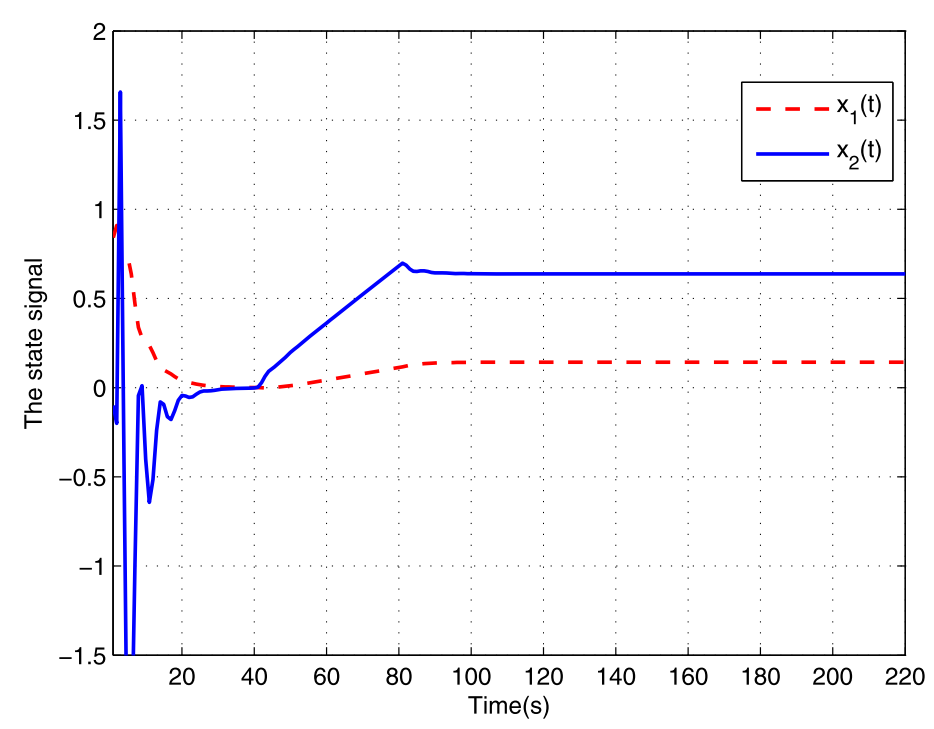

Figure 9 State curve of system (19) under delay-independent control design with target tracking.

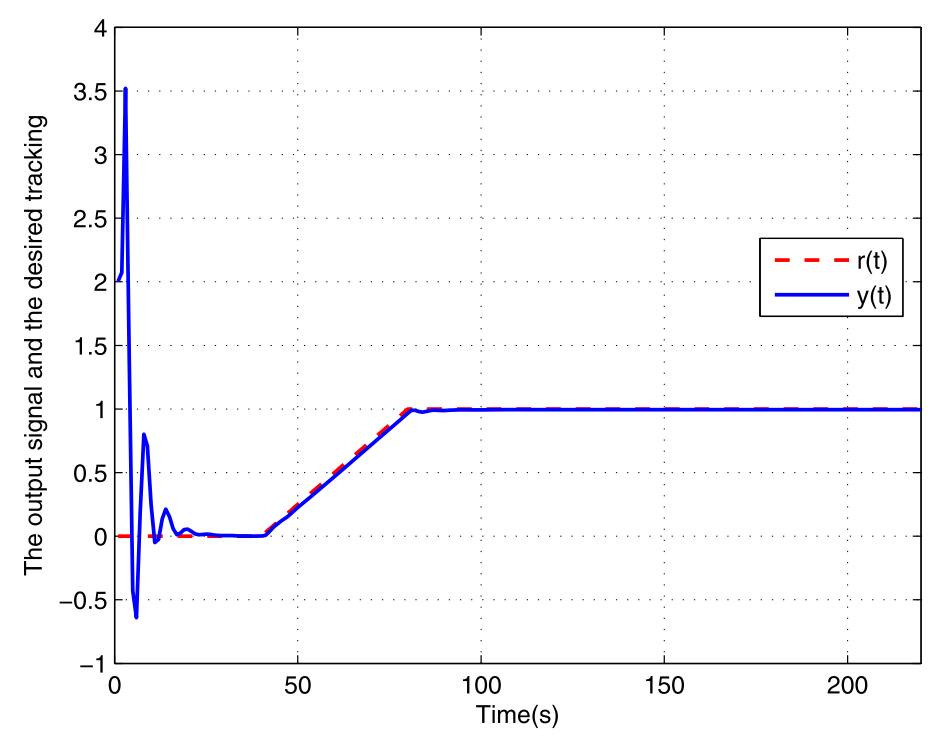

Figure 10 Output response of system (19) under delay-independent control design with target tracking.

\section{Conclusions}

In this paper, control design based on target tracking is investigated. The properties of the desired output are taken to construct an augmented error system. Based on the asymptotic stability theory of the nominal system, we present the feedback control for the augmented error system. The corresponding control design, which contains integrator on the tracking error signal, is deduced for the original system in Theorems 3 and 5. Two examples dealing with different kinds of desired output have been given to show the effectiveness of the proposed method. Control design in the paper contains not only the state feedback, but also the integrator on the tracking error signal, the integrals of the state and the de- 


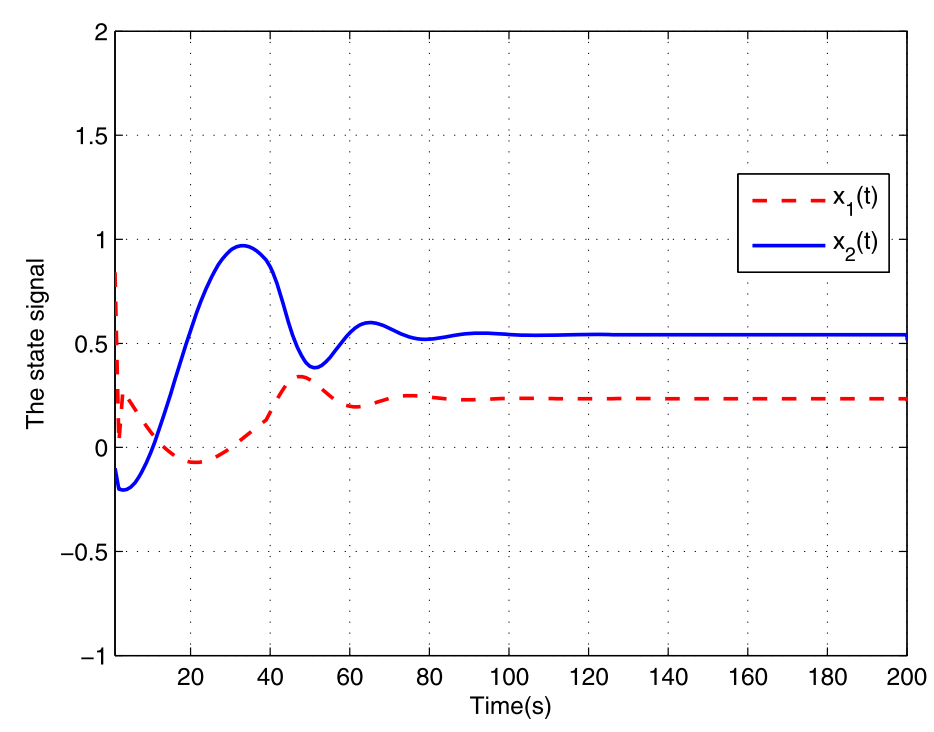

Figure 11 State curve of system (19) under delay-dependent control design with target tracking.

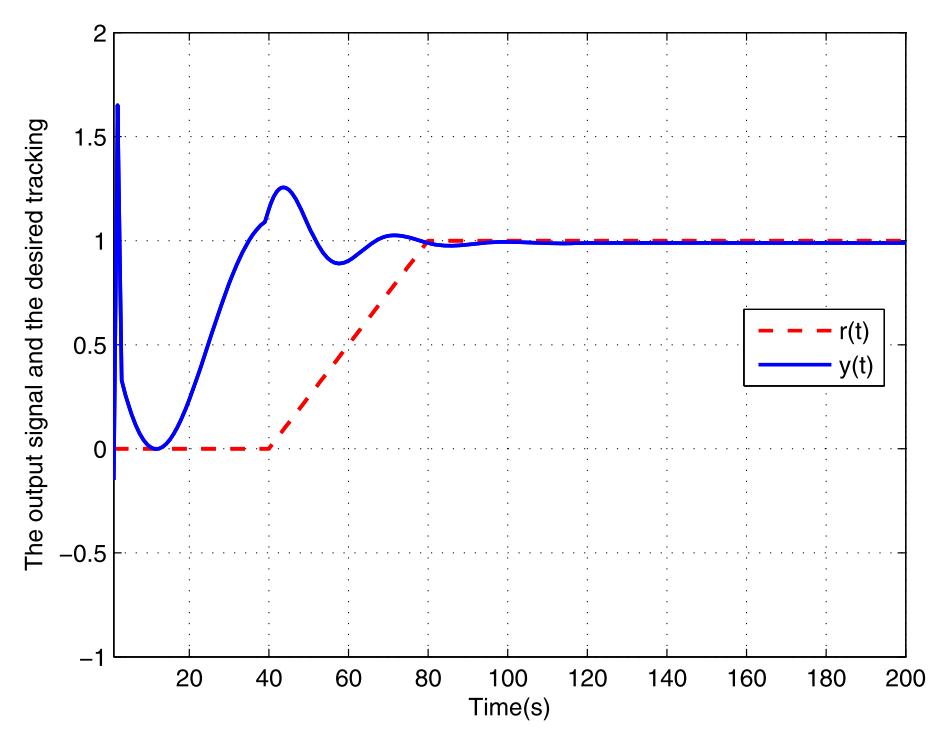

Figure 12 Output response of system (19) under delay-dependent control design with target tracking.

sired output. We hope that the proposed method in the paper can provide an approach to control design for a class of neutral systems described as system (1).

Competing interests

The authors declare that they have no competing interests.

Authors' contributions

The idea of this paper was proposed by $Y X$ and FL. The main theory was proved by $Y X$ and JZ. The paper was typed by $Y X$ and JZ. All authors read and approved the final manuscript. 


\section{Author details}

'Department of Basic Courses, Beijing Union University, No. 97 Beisihuan East Road, Chaoyang District, Beijing, 100101, P.R. China. ${ }^{2}$ School of Mathematics and Physics, University of Science and Technology Beijing, No. 30 Xueyuan Road, Haidian District, Beijing, 100083, P.R. China.

\section{Acknowledgements}

The work was supported by 'New Start' Academic Research Projects of Beijing Union University (Grant No. Zk10201503), the Importation and Development of High-Caliber Talents Project of Beijing Municipal Institutions (Grant No. IDHT201304089). We are indebted to Professor Wansheng Tang of Tianjin University for many useful comments. We are grateful to the anonymous reviewers for helpful advices that improved the presentation of the article.

\section{Received: 10 February 2015 Accepted: 6 August 2015 Published online: 03 September 2015}

\section{References}

1. Yamazaki, T, Hagiwara, T: Conversion of linear time-invariant time-delay feedback systems into delay-differential equations with commensurate delays. Int. J. Inf. Syst. Sci. 45, 1643-1656 (2014)

2. Lee, TH, Park, M-J, Park, JH, Kwon, O-M, Lee, S-M: Extended dissipative analysis for neural networks with time-varying delays. IEEE Trans. Neural Netw. Learn. Syst. 25, 1936-1941 (2014)

3. Chawda, V, OMalley, MK: Position synchronization in bilateral teleoperation under time-varying communication delays. IEEE/ASME Trans. Mechatron. 20, 245-253 (2015)

4. Aleksandrov, AY, Hu, G-D, Zhabko, AP: Delay-independent stability conditions for some classes of nonlinear systems. IEEE Trans. Autom. Control 59, 2209-2214 (2014)

5. Balasubramaniam, P, Krishnasamy, R, Rakkiyappan, R: Delay-dependent stability of neutral systems with time-varying delays using delay-decomposition approach. Appl. Math. Model. 36, 2253-2261 (2012)

6. Liu, S, Xiang, Z: Exponential $H_{\infty}$ output tracking control for switched neutral system with time-varying delay and nonlinear perturbations. Circuits Syst. Signal Process. 32, 103-121 (2013)

7. Kolmanovskii, VB, Myshkis, A: Applied Theory of Functional Differential Equations. Kluwer Academic, Dordrecht (1992)

8. Bellen, A, Guglielmi, N, Ruehli, AE: Methods for linear systems of circuit delay differential equations of neutral type. IEEE Trans. Circuits Syst. I, Fundam. Theory Appl. 46, 212-216 (1999)

9. Li, H: Robust Control for Neutral Delay Systems. Northwestern Polytechnical University Press, Xi'an (2006)

10. Pepe, P, Verriest, El: Lyapunov criteria for stability in $L_{p}$ norm of special neutral systems. Automatica 48, 132-138 (2012)

11. Qian, W, Liu, J, Sun, Y-X, Fei, S-M: A less conservative robust stability criteria for uncertain neutral systems with mixed delays. Math. Comput. Simul. 80, 1007-1017 (2010)

12. Chen, H: New criteria on stability analysis for uncertain neutral system with mixed time-varying delays. Optim. Control Appl. Methods 34, 487-501 (2013)

13. Ramakrishnan, K, Ray, G: Robust stability criteria for uncertain neutral systems with interval time-varying delay. J. Optim. Theory Appl. 149, 366-384 (2011)

14. Chen, Y, Zheng, W, Xue, A: A new result on stability analysis for stochastic neutral systems. Automatica 46, 2100-2104 (2010)

15. Kwon, OM, Park, MJ, Park, JH, Lee, SM, Cha, EJ: New delay-partitioning approaches to stability criteria for uncertain neutral systems with time-varying delays. J. Franklin Inst. 349, 2799-2823 (2012)

16. Zhang, D, Li, Y: $H_{\infty}$ filtering for linear neutral systems with mixed time-varying delays and nonlinear perturbations. J. Franklin Inst. 347, 1374-1390 (2010)

17. Zhong, G, Xu, Z, Li, X:H-infinite output tracking control for neutral systems with time-varying delay and nonlinear perturbations: a less conservative result. Appl. Mech. Mater. 48-49, 579-583 (2011)

18. Xia, J, Park, JH, Lee, TH, Zhang, B: $\mathcal{H}_{\infty}$ tracking of uncertain stochastic time-delay systems: memory state-feedback controller design. Appl. Math. Comput. 249, 356-370 (2014)

19. Wu, L-B, Yang, G-H: Robust adaptive fault-tolerant tracking control of multiple time-delays systems with mismatched parameter uncertainties and actuator failures. Int. J. Robust Nonlinear Control (2014). doi:10.1002/rnc.3241

20. Tsuchiya, T, Egami, T: Newest Automatic Control Technique: Digital Preview and Predictive Control. Beijing Science and Technology Press, Beijing (1994)

21. Katayama, T, Hirono, T: Design of an optimal servomechanism with preview action and its dual problem. Int. J. Control 45, 407-420 (1987)

22. Xu, Y, Liao, F: Preview control for a class of time-varying discrete systems with input time-delay. Control Decis. 28 466-470 (2013)

23. Zhao, G: Signal Analysis and Processing, 2nd edn. China Machine Press, Beijing (2006)

24. Wei, G, Wang, Z, Shu, H, Fang, J: Delay-dependent stabilization of stochastic interval delay systems with nonlinear disturbances. Syst. Control Lett. 56, 623-633 (2007) 